\title{
Surinformation : Nous devons améliorer le rapport signal-bruit
}

\author{
par Richard S Slavik
}

$\mathrm{L}$ 'édition anglaise de Wikipédia définit la surinformation comme un « excès d'information compliquant le traitement et l'assimilation pour les utilisateurs parce que parfois nous ne pouvons juger de la validité de la source d'information, ... et du risque de désinformation. [La surinformation] est un symptôme de l'ère de la haute technologie, où un être humain est incapable de tout assimiler dans cet univers où la population et la technologie sont en constante expansion ${ }^{1}$. " Cette infobésité est attribuable à un accès plus facile à l'information, à la surmultiplication de nouvelle information, à l'accroissement des moyens de reproduction et de diffusion de l'information, aux contradictions et aux inexactitudes de l'information, et à l'absence apparente de méthodes normalisées pour comparer et traiter l'information, ce qui aboutit nécessairement à " l'infopollution" et à un faible " rapport signal-bruit " ${ }^{1}$.

Les pharmaciens sont perçus par le public et les autres professionnels de la santé comme des experts en santé et en information sur les médicaments, capables de guider les patients dans un univers de données hasardeux, leur donnant un éclairage unique, les aidant à discriminer et leur exposant de façon pratique les risques, les bienfaits et la valeur des médicaments dans la prévention et le traitement des maladies et l'amélioration des soins aux patients. En revanche, il devient de plus en plus difficile d'accomplir cette tâche, car les pharmaciens sont bombardés de messages qui matraquent les vertus de médicaments " miracles ", les " dangers imminents » de réactions et d'interactions médicamenteuses nouvelles, et de " métamorphoses " des modes de vie au goût du jour ${ }^{2}$. Si nous ne reconnaissons pas l'impact de ce problème, n'appliquons pas de stratégies d'adaptation éprouvées et efficaces, et ne cherchons pas de solutions novatrices pour résister à ce flot d'information, les données mêmes sur lesquelles nous nous appuyons pour faire la promotion de traitements sûrs, efficaces et rentables, nous empêcheront d'agir.

Aujourd'hui, l'information sur les médicaments est plus accessible que jamais, et nous pouvons disposer de quantités phénoménales d'information en quelques secondes. D'ailleurs, une recherche récente dans PubMed sur le mot "drug " a produit plus de 3,4 millions d'articles. En limitant la recherche à des articles en anglais concernant les humains, on obtenait des chiffres toujours étonnants : 5320 études comparatives à répartition aléatoire, 610 méta-analyses, et 165 guides de pratique clinique! Cependant, cette information relativement de haute qualité, qu'on peut retracer par des recherches sélectives contrôlées, n'est pas ce qui met en péril le " rapport signal-bruit ». C'est plutôt l'existence d'une panoplie de services d'indexation et de résumés analytiques, de sites Web d'information sur les médicaments et de rapports par les médias de masse qui est responsable de la reproduction effrénée de l'information diffusée; de plus, l'information qui nous est « lancée " peut être rapportée, interprétée ou utilisée de façon incomplète, imprécise ou incorrecte ${ }^{3}$. Pensez seulement au nombre de nouveaux rapports contradictoires dont vous avez entendu parler sur les effets cliniques de la caféine, du vin rouge, du chocolat, des suppléments de vitamines, émanant souvent d'études peu rigoureuses, dont les résultats ont été mal rapportés ou appliqués. Les renseignements sur les médicaments d'ordonnance ajoutent un autre niveau de complexité et de confusion, contribuant à la perte du « signal » dans le champ du " bruit ". Cette situation soulève des questions urgentes de patients confus et de professionnels de la santé agités qui, bien trop souvent, demandent à ce qu'on modifie le traitement médicamenteux avant même d'avoir évalué l'information erronée sur laquelle les rapports sont fondés.

Lapproche de la médecine fondée sur des données probantes nous aidera à naviguer dans cet océan d'information, et les pharmaciens doivent prendre la responsabilité d'utiliser leurs connaissances, leurs compétences, leur attitude et leur comportement afin d'évaluer le "signal » dans tout ce « bruit ». Nous devons être déterminés à poser des questions ciblées, à retrouver les données les plus probantes, à évaluer les résultats importants sur le plan clinique, à faire une évaluation critique des données pour voir si on peut se fier aux résultats, à comprendre l'importance des risques, des bienfaits et des coûts et à déterminer si et comment on peut appliquer les résultats à nos patients. Alors que le temps nous bouscule toujours plus, 
nous sommes tentés de prendre le chemin le plus facile, pour simplement suivre le courant de l'opinion publiée dominante. Mais nous devons résister, et nous évertuer à évaluer le "signal " plutôt que d'agir sous l'emprise du " bruit ».

Nous avons aussi besoin de meilleurs systèmes pour nous appuyer. En effet, lorsque des articles sont soumis à des revues comme le JCPH, puis publiés par celles-ci, leur "signal " sera amplifié par de multiples canaux de reproduction et de diffusion. Les comités de rédaction de telles revues doivent par conséquent assurer la qualité de la méthodologie des études et de l'exactitude des rapports. En outre, les limites de la recherche doivent être signalées, les résultats doivent être interprétés dans le contexte de toutes les données disponibles, et les conclusions et recommandations ne doivent pas être exagérées.

Plus tôt cette année, le JCPH a pris des mesures supplémentaires pour appuyer les auteurs, les rédacteurs et les réviseurs à minimiser les biais et le " bruit " dans les articles de recherche, en mettant à disposition une variété d'outils de recherche conçus pour améliorer la qualité de la méthodologie et l'exactitude des rapports des méta-analyses (QUOROM [QUality Of Reporting Of Meta-analyses]), des études comparatives à répartition aléatoire (CONSORT [Consolidated Standards of Reporting Trials]), des études comparatives sans répartition aléatoire (TREND [Transparent Reporting of Evaluations with Nonrandomized Designs]), et des études d'observation (STROBE [STrengthening the Reporting of OBservational studies in Epidemiology] $)^{4}$. Fait intéressant, la déclaration QUOROM a été mise à jour et bonifiée dernièrement et sera bientôt publiée sous le nouveau nom PRISMA (Preferred Reporting of Systematic Reviews and Meta-Analyses) $)^{5}$.

Par ailleurs, le JCPH favorise les discussions ouvertes sur les questions de controverse cliniques par le truchement de sa chronique "Le pour et le contre ", une tribune pour faire connaître l'ensemble des données probantes et proposer un débat mieux éclairé sur d'importants sujets, et rendre le "signal » plus clair. Dans ce numéro du Journal, Le pour et le contre traite de la controverse entourant le prolongement de la fenêtre pour la thrombolyse dans les cas d'accident vasculaire cérébral ischémique aigu, de 3 à 4,5 heures ${ }^{6,7}$. Les médias de masse et les rapports à l'intention des professionnels de la santé ont presque partout souscrit aux résultats de l'étude ECASS III («European Cooperative Acute Stroke Study III ") ${ }^{8}$ et ont peut-être actualisé trop rapidement leurs guides de pratique clinique, avec étonnamment une publication très timide des points de vue franchement contradictoires. Par exemple, un article dans Medscape intitulé "ECASS 3 [sic] gets a warm welcome from the stroke community ", publié en ligne au début d'octobre 2008, a cité huit médecins, tous ayant des commentaires positifs à l'endroit de l'étude ECASS III et étant favorables au prolongement de la fenêtre pour la thrombolyse'. Bien que cet article ne représente pas nécessairement le « signal » complet sur cette question, il exerce cependant une influence considérable. Dans leur enthousiasme, les comités de pharmacologie et de thérapeutique ainsi que les concepteurs d'algorithmes cliniques et d'ordonnances préimprimées n'ont peut-être pas pris de temps pour évaluer d'un œil critique toutes les données pertinentes et pour tenir compte de l'ensemble des risques et des bienfaits de la prolongation de la fenêtre pour la thrombolyse. Nous espérons que le JCPH et sa chronique « Le pour et le contre " continueront de servir de tribune aux pharmaciens et de répondre à des questions comme celle-ci, et aussi de les inciter à prendre le temps de remettre les réponses en question. Cette tribune devrait contribuer à rendre le « signal » de l'information sur les médicaments plus clair dans ce "bruit " incessant qui nous empêche de plus en plus de faire la promotion de traitements sûrs, efficaces et rentables pour nos patients.

\section{References}

1. Information overload. Dans : Wikipedia, the free encyclopedia. San Francisco (CA) : Wikimedia Foundation Inc; [aucune date]. Publié à en.wikipedia.org/wiki/Information_overload. Consulté le 2 mars 2009.

2. Cassels A, Hughes MA, Cole C, Mintzes B, Lexchin J, McCormack JP. Drugs in the news: an analysis of Canadian newspaper coverage of new prescription drugs. CMAJ 2003;168(9):1133-1137.

3. Therapeutics Initiative. Evaluating the media as a source of drug therapy information. Ther Lett 2007 Nov-Dec;67. Publié à www.ti.ubc.ca/ $\mathrm{PDF} / 67$.pdf.

4. Research tools for authors, editors, and reviewers. Ottawa $(\mathrm{ON})$ : Société canadienne des pharmaciens d'höpitaux; juin 2008. Publié à cshp.ca/dms/ dmsView/1_CJHPREsearchToolsJune2008.pdf. Consulté le 2 mars 2009.

5. Moher D. Producing clear, accurate and transparent reports of systematic reviews: an attainable goal [éditorial]. Dans : BMJ clinical evidence [Internet]. Londres (R.-U.) : BMJ Publishing Group; 20 octobre 2008. Publié à clinicalevidence.bmj.com/downloads/20-10-08.pdf. Consulté le 2 mars 2009.

6. Patel T. Should the window for stroke thrombolysis be extended to 4.5 hours? The "pro" side. Can J Hosp Pharm 2009;62(3):248-249.

7. Loewen PS. Should the window for stroke thrombolysis be extended to 4.5 hours? The "con" side. Can J Hosp Pharm 2009;62(3):250-251.

8. Hacke W, Kaste M, Bluhmki E, Brozman M, Davalos A, Guidetti D, et al.; ECASS Investigators. Thrombolysis with alteplase 3 to 4.5 hours after acute ischemic stroke. N Engl J Med 2008;359(13):1317-1329.

9. Jeffrey S. ECASS 3 gets a warm welcome from the stroke community (Vienna, Austria). Dans : Medscape Medical News. New York (NY) : Medscape, LLC; octobre 2008. Publié à www.medscape.com/viewarticle/581053 (inscription nécessaire pour accéder au contenu). Consulté le 2 mars 2009.

Richard S Slavik, B. Sc. Pharm., ACPR, Pharm. D., FCSHP travaille auprès de la Regional Pharmacy, Interior Health Authority, Kelowna, ColombieBritannique. Il est également rédacteur adjoint du JCPH.

\section{Adresse de correspondance :}

Dr Richard S Slavik

Regional Pharmacy

Interior Health Authority

200-1835 Gordon Drive

Kelowna (C.-B.)

V1Y $3 \mathrm{H} 5$

Courriel : richard.slavik@interiorhealth.ca 